\title{
ORIGINAL ARTICLE NAP (davunetide) rescues neuronal dysfunction in a Drosophila model of tauopathy
}

\author{
S Quraishe, CM Cowan and A Mudher
}

\begin{abstract}
Alzheimer's disease (AD) is a devastating neurodegenerative disease causing irreversible cognitive decline in the elderly. There is no disease-modifying therapy for this condition and the mechanisms underpinning neuronal dysfunction and neurodegeneration are unclear. Compromised cytoskeletal integrity within neurons is reported in AD. This is believed to result from loss-of-function of the microtubule-associated protein tau, which becomes hyper-phosphorylated and deposits into neurofibrillary tangles in AD. We have developed a Drosophila model of tauopathy in which abnormal human tau mediates neuronal dysfunction characterised by microtubule destabilisation, axonal transport disruption, synaptic defects and behavioural impairments. Here we show that a microtubule-stabilising drug, NAPVSIPQ (NAP), prevents as well as reverses these phenotypes even after they have become established. Moreover, it does not alter abnormal tau levels indicating that it by-passes toxic tau altogether. Thus, microtubule stabilisation is a disease-modifying therapeutic strategy protecting against tau-mediated neuronal dysfunction, which holds great promise for tauopathies like AD.
\end{abstract}

Molecular Psychiatry (2013) 18, 834-842; doi:10.1038/mp.2013.32; published online 16 April 2013

Keywords: Alzheimer's disease; axonal transport; Drosophila; microtubules; NAP (davunetide)

\section{INTRODUCTION}

Although Alzheimer's disease (AD) was first described over 100 years ago, the neurodegenerative mechanisms underpinning this disease remain unclear. Thus, disease-modifying therapies still elude us and treatment is restricted to provision of symptomatic relief. ${ }^{1}$ One avenue of research investigates how hyper-phosphorylated tau, a microtubule-associated protein forming the tangle pathology in $A D$, causes neuronal dysfunction and degeneration. Hyper-phosphorylated tau cannot effectively bind and stabilise microtubules. It is hypothesised that this compromises cytoskeletal integrity within affected neurons in $\mathrm{AD}^{2-4}$ disrupting axonal transport and synaptic function ${ }^{5,6}$ and manifesting in clinical symptoms. ${ }^{7}$ Compromised and reduced numbers of microtubules are reported in post-mortem AD brains. ${ }^{8}$ Furthermore, a direct correlation between hyper-phosphorylated tau-mediated axonal transport disruption and behavioural impairment is demonstrated in Drosophila (htau ${ }^{\mathrm{ON} 3 \mathrm{R}}$ ) and rodent (tau-T44 tg mice) models of $A D{ }^{6,9}$ Supporting this, tangle pathology closely correlates with cognitive decline in $A D$ patients. ${ }^{10}$ Hence, tau-based therapies are being considered for AD. ${ }^{11,12}$ Current strategies include drugs to reduce tau phosphorylation and aggregation. Recently, the use of microtubulestabilising drugs to compensate for tau loss-of-function has been explored. ${ }^{13}$ Paclitaxel, a microtubule-stabilising drug, reduces axonopathy and improves behaviour in a rodent model of tauopathy. ${ }^{14}$ Although this supports the idea of by-passing pathogenic tau by directly stabilising microtubules, the high toxicity and poor blood-brain barrier (BBB) permeability of such drugs make them unsuitable for treating patients. This prompted the development of a new generation of microtubule-stabilising agents exhibiting low toxicity and high BBB permeability. ${ }^{15,16}$

The small octapeptide NAPVSIPQ (NAP), derived from activitydependent neuroprotective protein, ${ }^{15}$ interacts with and stabilises neuron-specific $\beta$ III-tubulin in vitro. ${ }^{17}$ Importantly, NAP penetrates the BBB and is non-toxic. ${ }^{18,19}$ The neuroprotective activities of NAP are documented in many in vivo and in vitro models. ${ }^{16}$ Studies in rodent models of $A D$, suggest that NAP's protective capacity is mediated by its ability to stabilise the microtubule cytoskeleton. ${ }^{20-22}$ However, this is yet to be demonstrated in vivo.

Here we show that NAP by-passes abnormal tau and compensates for tau loss-of-function thus protecting against neuronal dysfunction in a Drosophila model of tauopathy. This model is ideally suited for this investigation because the phenotypes arise as a consequence of abnormal tau-mediated cytoskeletal destabilisation in the absence of tau aggregation. ${ }^{4-6}$

\section{MATERIALS AND METHODS}

\section{Stocks and drug treatment}

Expression of htau ${ }^{\text {ON3R }}$ (Bloomington Stock Centre, Bloomington, IN, USA; stock no. 181), htau ${ }^{\mathrm{N} 4 \mathrm{R}}$ (provided by Dr E Skoulakis; Alexander Fleming Institute, Athens, Greece) and amyloid beta-42 (A $\left.\beta_{42}\right): A \beta_{42} ;$ htau ${ }^{\mathrm{ON} 4 \mathrm{R}}$ (provided by Dr D Crowther; University of Cambridge, Cambridge, UK) was directed to Drosophila melanogaster motor neurons using either the motor neuron-specific drivers D42-Gal4, or the D42-Gal4 driver recombined with green fluorescent protein (GFP)-tagged neuropeptide-Y (D42-GAL4.UAS-NPY:GFP) ${ }^{6}$ (UAS-NPY:GFP provided by $\mathrm{Dr}$ I Robinson, University of Cambridge). Pan-neural expression was achieved with the Elav-Gal4 driver (Bloomington Stock Centre; stock no. 458). NAP (Peptide Protein Research, Fareham, UK) was delivered to basic fly food at concentrations of $50 \mathrm{ng} \mathrm{ml}^{-1}, 500 \mathrm{ng} \mathrm{ml}^{-1}, 2.5 \mathrm{\mu g} \mathrm{ml}^{-1}$ or $25 \mathrm{\mu g} \mathrm{ml}^{-1}$.

Larval locomotion and electron microscopy analysis were conducted as previously described. ${ }^{4,23}$

\section{In vivo axonal transport analysis}

Treatment groups were subjected to timed lays on apple juice agar plates. F1 eggs were transferred to basic or NAP food. Htau ${ }^{\text {ONR }}$ larvae were assessed 
at $\mathrm{L} 3$ wandering stage (day 5) at $23^{\circ} \mathrm{C}$. $\mathrm{Htau}^{\mathrm{ON} 4 \mathrm{R}}$ and $\mathrm{A} \beta_{42} ;$ ihtau ${ }^{\mathrm{ON} 4 \mathrm{R}}$ larvae were reared at $29^{\circ} \mathrm{C}$. For the NAP rescue experiment, early L3 larvae were removed from food plates at day 4 and either subjected to axonal transport analysis or moved onto a basic food plate or a NAP food plate for $24 \mathrm{~h}$. Axonal transport was analysed as previously described. ${ }^{6,24}$

\section{Immunohistochemistry}

Dissected L3 larvae were immunostained as previously described. ${ }^{5}$ Body walls were incubated in goat anti-horseradish peroxidase conjugated to fluorescein isothiocyanate (1:1000; ICN/Cappel, Solon, $\mathrm{OH}$, USA) and mouse anti-tubulin (1:500; E7-Developmental Hybridoma Bank, lowa City, IA, USA) antibodies and with Alexa Fluor 594 donkey anti-mouse (1:200; Invitrogen, Paisley, UK) secondary antibody. Muscle 4 from abdominal segments A3-A7 was imaged. ${ }^{5}$

\section{Microtubule-binding assay}

Three heads of 1 to 3-day-old flies, or 10 larval (L3) ventral cords were pooled and homogenised in 30 and $40 \mu \mathrm{l}$, respectively, of microtubulebinding assay buffer as previously described. ${ }^{4}$

\section{Immunopreciptiation assay}

Ten heads of 1 to 3-day-old flies were homogenised in $500 \mu l$ of microtubule-binding assay buffer and interactions between htau ${ }^{\mathrm{N} 3 \mathrm{R}}$ and dtau were assessed as described previously. ${ }^{4}$

\section{Western blotting}

Adult heads or dissected L3 larval ventral cords were homogenised, resolved by sodium dodecyl sulfate-polyacrylamide gel electrophoresis. Blots were probed with anti-human tau (1:15,000, Dako, Cambridge, UK), anti-dtau (1:500, Professor St Johnston, University of Cambridge), anti-phospho-tau: PHF-1 (1:2000, Dr Peter Davies, Albert Einstein College of Medicine, Bronx, NY, USA), AT180 (1:100, Biosciences, Nottingham, UK), AT8 (1:800, Biosciences), tau-1 (1:2000, Millipore, Watford Herts, UK), pS262 (1:1000, Invitrogen), antitubulin (1:200) and anti-kinesin (1:5000, Cytoskeleton, Denver, CO, USA). Signal was detected using fluorescently conjugated secondary antibodies.

\section{Statistics}

Statistical analysis was carried out using Prism 5.0 (GraphPad, University of Southampton, Southampton, UK). T-test or one-way analysis of variance, with Bonferroni's Multiple comparison post-hoc analysis.

\section{RESULTS}

NAP prevents htau ${ }^{\mathrm{ON} 3 \mathrm{R}}$-mediated disruption of locomotor behaviour

Expression of the highly phosphorylated 0N3R human tau isoform $\left(\right.$ htau $\left.{ }^{\mathrm{ON} 3 \mathrm{R}}\right)$ in motor neurons of Drosophila manifests in a number of distinct phenotypes including crawling defects in larvae. ${ }^{6}$ We first tested whether this phenotype could be prevented by NAP. Untreated htau ${ }^{\text {ON3R }}$ larvae exhibited a restricted and noncontinuous crawling behaviour (Figure 1b; Supplementary Video 1) compared with controls (Figure 1a; Supplementary Video 2). This defective crawling behaviour improved significantly following NAP treatment leading to smoother, more directed movement over a greater distance like controls (Figure 1c; Supplementary Video 3). Using the image-tracking software Ethovision, crawling parameters were quantified including body wall contractions, velocity and meander (turning rate per distance travelled). In all, $2.5 \mu \mathrm{g} \mathrm{ml}^{-1} \mathrm{NAP}$ significantly improved body wall contractions and velocity compared with un-treated htau ${ }^{\text {N3R }}$ larvae (Figures $1 \mathrm{~d}$ and e). Meander was also improved, but did not reach significance $(P=0.06)$ (Figure 1f). Crawling behaviour of controls treated with NAP was no different to untreated controls (Supplementary Figure 1).

NAP stabilises microtubules and improves axonal transport and neuromuscular junction (NMJ) morphology

As disruption of the microtubule cytoskeleton causes behavioural defects in this model, we investigated whether microtubule stabilisation was the mechanism by which NAP rescued the locomotor phenotype of htau ${ }^{\text {N } 3 R}$ larvae. The ultrastructural organisation of the microtubule cytoskeleton was examined in transverse sections of peripheral nerves by transmission electron microscopy. Controls displayed characteristic, $25 \mathrm{~nm}$ circular transverse microtubule profiles (black arrows Figures 2a-c). In contrast, htau ${ }^{\mathrm{N} 3 \mathrm{R}}$ larvae had noticeably fewer such microtubule profiles and displayed many disorganised and mis-aligned microtubules (black arrowheads Figures $2 d-f$ ). A number of axons relatively devoid of microtubules were also evident in htau ${ }^{\text {N } 3 R}$ larvae (asterisks Figure 2f). NAP re-stabilised the disrupted and disorganised microtubules as evidenced by the appearance of more intact transverse microtubule profiles in treated htau ${ }^{\text {NN3R }}$ larvae (black arrows Figures $2 \mathrm{~g}-\mathrm{i}$ ). Quantification of the number of intact microtubule profiles per axon confirmed that there were significantly fewer microtubule profiles in htau ${ }^{\text {ON } 3 R}$ axons $(5.32 \pm 0.28)$ compared with controls $(8.10 \pm 0.20)$. NAP increased this number back to control levels $(8.07 \pm 0.30$; Figure $2 \mathrm{j})$. The range of intact microtubules per individual axon in each condition is illustrated in Figure 2k. Almost half the axons from controls contained large numbers of microtubules $(>5)$, with very few axons containing no microtubules (1\%). However, the majority $(\sim 65 \%)$ of htau ${ }^{\mathrm{ON} 3 \mathrm{R}}$ axons contained either none $(30 \%)$ or very few $(<5)$ microtubules $(35 \%)$. In contrast, the peripheral nerves of NAP-treated htau ${ }^{\mathrm{N} 3 \mathrm{R}}$ larvae had a similar microtubule profile to controls. There was an increase in the number of axons containing large numbers $(>5)$ of intact transverse microtubules (58\%) compared with untreated htau ${ }^{\mathrm{ON} 3 \mathrm{R}}(35 \%)$, and a reduction in the number of axons with no microtubules (7\%; Figure 2k). NAP treatment did not change the number of microtubule profiles in controls (untreated controls had $9.22 \pm 0.42$ intact microtubule (MT) profiles whereas NAP-treated controls had $9.98 \pm 0.45$ intact MT profiles; Supplementary Figure 2).

We next investigated whether the restoration of microtubule integrity in NAP-treated htau ${ }^{\mathrm{N} 3 \mathrm{R}}$ larvae improves axonal transport as we know that axonal transport is disrupted in this model as a consequence of microtubule breakdown. ${ }^{6}$ Expression of vesicular neuropeptide-Y-GFP (vGFP) in motor neurons of larvae allows this htau ${ }^{\mathrm{N} 3 \mathrm{R}}$-mediated phenotype to be visualised in vivo, in real time, in living intact larvae. A homogeneous distribution of vGFP indicating efficient axonal transport was evident in motor neurons of NAP-treated and untreated controls (Figures 3b.i and a.i). As expected, in htau ${ }^{\text {NNR }}$ larvae the distribution of vGFP was dramatically different, with the appearance of large vGFP accumulates within axons, illustrating profound axonal transport disruptions (Figure 3c.i). However, in NAP-treated htau ${ }^{\text {ON3R }}$ larvae axonal transport appeared normal, with very few vGFP accumulates (Figure 3d.i). Quantification of the total area occupied by vGFP accumulates confirmed this result (Figure 3e). A 10-fold higher dose of NAP $\left(25 \mu \mathrm{g} \mathrm{ml}^{-1}\right)$ did not lead to any further improvement (Figure 3e). NAP also significantly reduced axonal transport defects in animals expressing comparable levels of another tau isoform (htau ${ }^{\mathrm{NN} 4 \mathrm{R}}$ alone or htau ${ }^{\mathrm{NN} 4 \mathrm{R}}$ and $\mathrm{A} \beta_{42}$ $\left(A \beta_{42} ;\right.$ htau $\left.\left.^{\mathrm{ON} 4 \mathrm{R}}\right)\right)$, illustrating that NAP can rescue defects induced by both 3 and 4 repeat tau isoforms (Figure $3 \mathrm{f}$ and Supplementary Figure 3).

Synaptic function and NMJ morphology are also compromised in our model. ${ }^{5}$ To ascertain whether the protective effect of NAP extends to the synapse, the NMJ on muscle 4 was visualised using an antibody against a neuronal surface antigen (anti-horseradish peroxidase). Anti-tubulin antibody was also used to visualise the microtubule cytoskeleton within the NMJ terminals. The NMJ morphology in controls was characterised by a bead-like distribution of pre-synaptic boutons (Figures 3a.ii-iv). The tubulin cytoskeleton extended into the distal ends of the NMJ (Figure 3a.iii). Neither the morphology nor the tubulin cytoskeleton of these controls was altered by NAP (Figure 3b.ii-iv). In contrast, htau ${ }^{\text {N3R }}$ larvae NMJs exhibited profound 

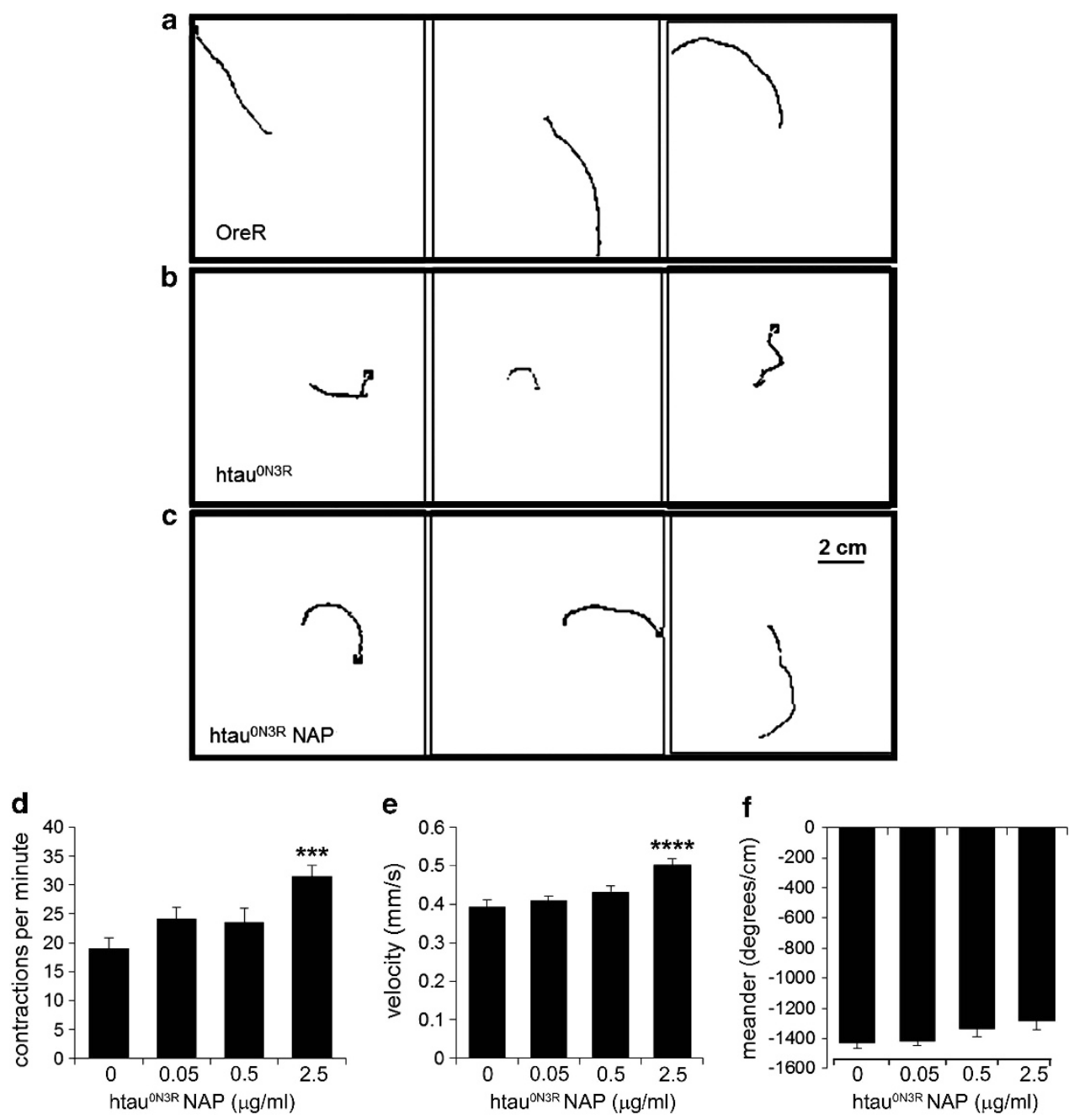

Figure 1. NAPVSIPQ (NAP) improves the locomotor phenotype in htau ${ }^{\text {ON3R }}$-expressing Drosophila. Oral administration of $2.5 \mu g \mathrm{ml}^{-1} \mathrm{NAP}$ improves the locomotor performance of htau ${ }^{\mathrm{NN} 3 \mathrm{R}}$ wandering third instar larvae. (a-c) Traces of paths taken by L3 larvae in 2-min free crawling on $100 \mathrm{~cm}^{2}$ plates are shown for OreR controls (a), htau ${ }^{\mathrm{ON} 3 R}$ untreated larvae (b) and htau ${ }^{\text {ON3R }}$ larvae treated with NAP (c). Crawling performance was quantified using a tracking-software Ethovision. In all, $2.5 \mu \mathrm{g} \mathrm{ml}^{-1}$ NAP improved crawling performance on three different measures: body wall contractions per minute (d), velocity (e) and meander (f). Scale bar: $2 \mathrm{~cm}$. Error bars represent mean \pm s.e.m., (d-f); oneway analysis of variance, post-hoc Bonferroni's multiple comparison test, ${ }^{* * *} P<0.001$ (body wall contractions); ${ }^{* * *} P<0.0001$ (velocity); $P=0.06$ (meander). $n=16-20$

morphological aberrations, including thinning of inter-bouton axon segments and appearance of 'mini' satellite boutons (arrowheads Figure 3c.ii). The cytoskeleton was also disrupted, with a complete absence of tubulin immunoreactivity in the most distal regions (asterisk Figure 3c.iii). NMJs of NAP-treated htau ${ }^{\text {N3R }}$ larvae also exhibited morphological aberrations similar to those of untreated htau ${ }^{\mathrm{N} 3 \mathrm{R}}$ larvae, but with a greater number of 'mini' satellite boutons (arrowheads Figure 3d.ii). However, the tubulin cytoskeleton in these animals extended into the most distal ends of the NMJ similar to that of the controls (asterisks Figure 3d.iii). The area encompassed by this NMJ was significantly smaller in htau ${ }^{\text {N } 3 R}$ larvae compared with controls. NAP treatment significantly increased this (Figure $3 \mathrm{~g}$ ).

Tau phosphorylation is not altered by NAP

Hyper-phosphorylation of htau in our model reduces its (and the endogenous Drosophila tau (dtau)'s) microtubule-binding ability thus compromising cytoskeletal integrity. ${ }^{4}$ We therefore asked whether NAP was preventing the emergence of the htau ${ }^{\text {ON3R }}$ mediated phenotypes by reducing tau phosphorylation.
We examined a number of phospho-tau epitopes associated with $A D$ in both NAP-treated and untreated htau ${ }^{\text {NN3 }}$ larvae and adults. NAP did not alter tau phosphorylation at any of the sites examined (Figures $4 a-d$ and Supplementary Figures $4 A-E$ ). Although there was a trend for reduced tau phosphorylation at thr231/235 (AT180) following NAP treatment, this was not statistically significant $(P=0.3409$; Figure $4 \mathrm{~b})$. As phospho-tau levels were unaltered, we next explored the possibility that NAP was protecting the htau ${ }^{\text {ON } 3 R}$ larvae by increasing endogenous $\mathrm{dtau}$ and/or by reducing toxic htau. Neither the total htau or dtau levels were altered by NAP in both larvae and adults, suggesting that NAP does not block or enhance degradation of these proteins (Figures $4 \mathrm{e}$ and f; Supplementary Figures $4 \mathrm{~F}$ and G). Interestingly, although dtau levels were unaltered by NAP, the percentage of dtau bound to MTs increased following NAP treatment in both htau ${ }^{\mathrm{N} 3 \mathrm{R}}$-expressing larvae and adults (Figures $4 \mathrm{~g}$ and $\mathrm{h}$ ). However, htau binding to MTs was not similarly increased (Figures $4 \mathrm{i}$ and $\mathrm{j}$ ). This subcellular redistribution of dtau to the MTs was not attributable to the reduction of pathological $\mathrm{htau}^{\mathrm{ON} 3 \mathrm{R}} / \mathrm{dtau}$ interaction that we have previously reported ${ }^{4}$ (Figure 4k). 

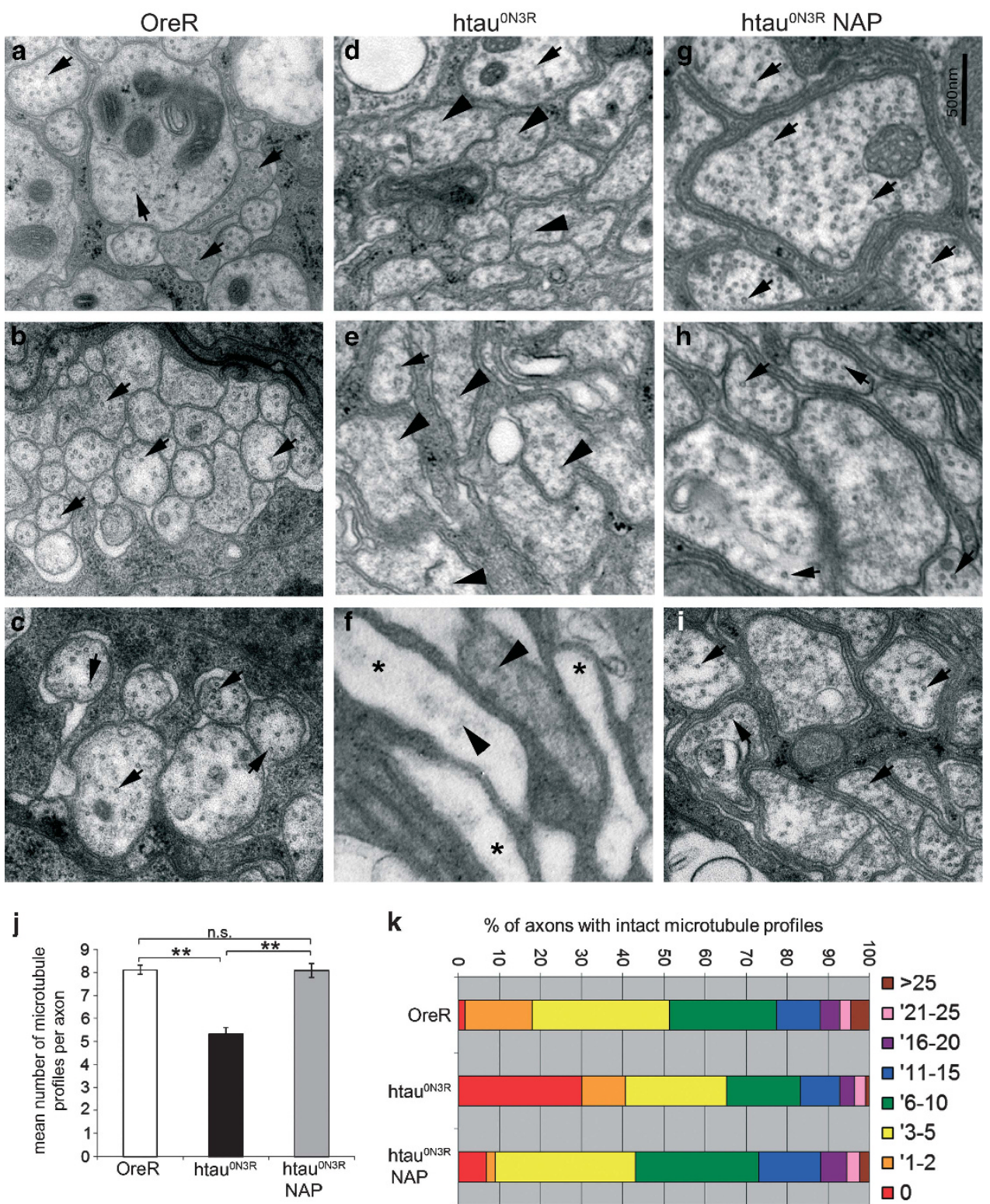

Figure 2. NAPVSIPQ (NAP) protects against microtubule destabilisation in htau ${ }^{\mathrm{ON} 3 \mathrm{R}}$ Drosophila. Representative electron micrographs showing transverse sections of peripheral nerves in L3 larvae. Axon profiles in control larvae show regularly spaced correctly aligned transverse microtubules (black arrows in a-c). In axons of htau ${ }^{\mathrm{ON} 3 \mathrm{R}}$ larvae, the microtubules were disrupted, with fewer correctly aligned transverse microtubules (black arrows in $\mathbf{d}-\mathbf{f}$ ) and more disorganised microtubules visible in the same axon profiles (black arrowheads in d-f). Axons relatively devoid of microtubules were also observed (asterisks in f). However, in htau ${ }^{\text {ON3R }}$ larvae fed $2.5 \mu \mathrm{g} \mathrm{ml^{-1 }} \mathrm{NAP}$, there was a complete rescue of microtubule integrity $(\mathbf{g}-\mathbf{i})$. Scale bar $500 \mathrm{~nm}$. Control axons contained on average $8.10 \pm 0.20$ correctly aligned microtubule profiles per cross-section; larvae-expressing htau ${ }^{\mathrm{ON} 3 \mathrm{R}}$ contained only $5.32 \pm 0.28$, and those expressing htau ${ }^{\mathrm{N} 3 \mathrm{R}}$ but reared on NAP contained $8.07 \pm 0.30$ profiles $(j)$. Average numbers of correctly aligned visible microtubule profiles in axons of control and htau ${ }^{\text {N3R }}$ NAP treated animals were significantly greater than those of untreated htau ${ }^{\mathrm{ON} 3 \mathrm{R}}$ animals. Axons exhibited a range of intact correctly aligned transverse microtubules (k): the majority of control axons contained large numbers of microtubules $(>5)$, and very few axons contained no microtubules. However, the majority of htau ${ }^{\text {N } 3 R}$ axons contain either none or very few microtubules $(<5)$. Htau ${ }^{\text {N } 3 R}$ larvae reared on NAP had a greater number of axons containing large numbers of intact transverse microtubules $(>5)$ and fewer axons with no microtubules (k). (Error bars represent mean \pm s.e.m., ${ }^{* *} P<0.01$, as determined by unpaired Student's two-tailed $t$-test, $n=5$ ). NS, not significant.

NAP not only prevents the emergence of tau phenotype, it also rescues it once established

The results thus far show that NAP prevents the emergence of hyper-phosphorylated tau-mediated neuronal and behavioural phenotypes. To investigate whether NAP can alter tau phenotypes if administered after they are already established, we devised a 'rescue protocol'. In this protocol, htau ${ }^{\text {ONBR }}$ larvae were reared on normal food for 4 days and then transferred to food containing
NAP for 1 day. Axonal transport was assessed at day 4 before NAP treatment was begun and then again at day 5, after only 1 day of NAP treatment. Axonal transport was disrupted in untreated htau $^{0 N 3 R}$ larvae even at day 4 (light grey bar Figure 5a). However, NAP treatment for 1 day significantly reduced this axonal transport disruption (white bar Figure 5a). Importantly, this rescue of axonal transport deficit following only 1 day of NAP treatment was the same as if it were given continuously for 5 days (striped 

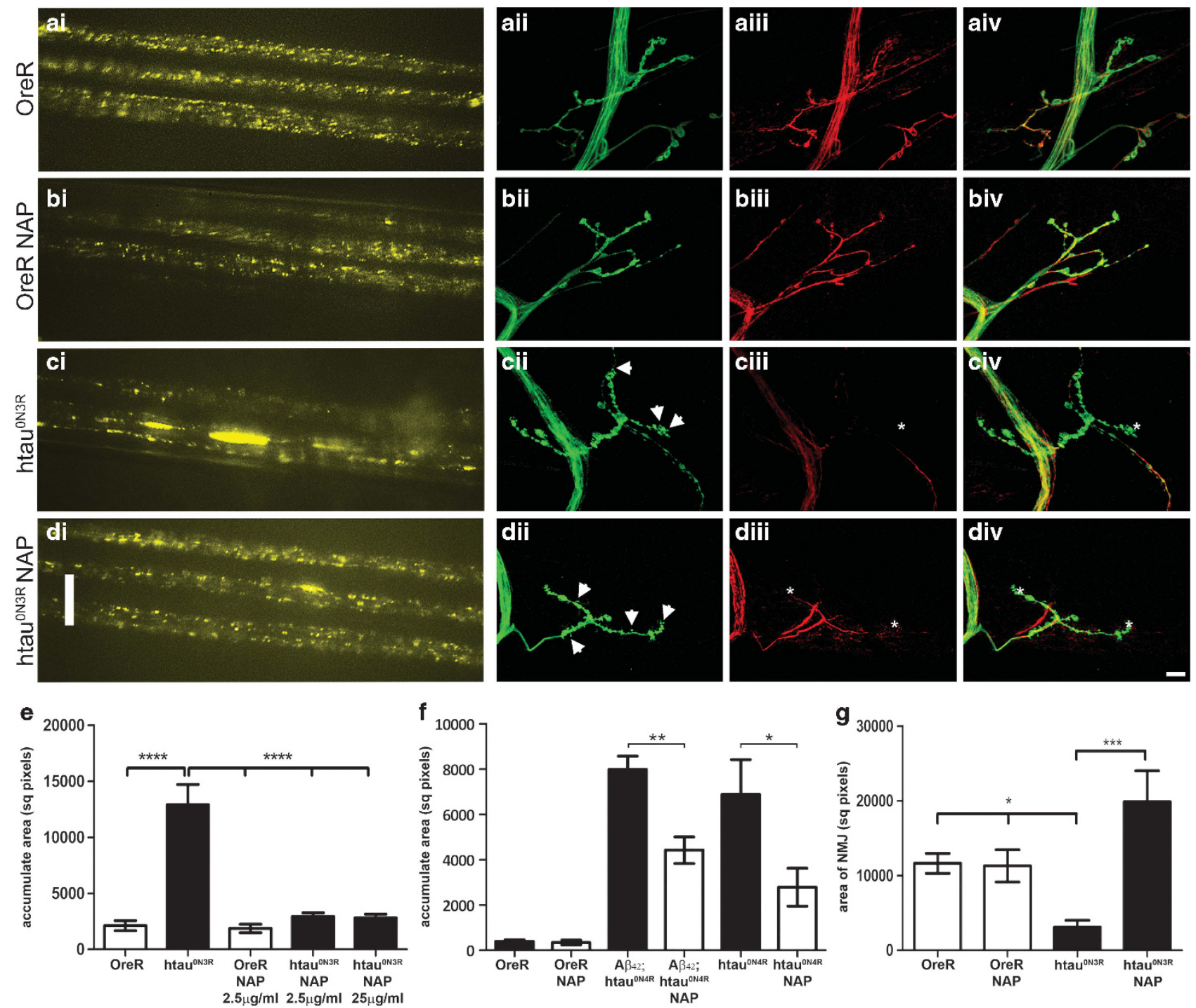

Figure 3. NAPVSIPQ (NAP) prevents axonal transport deficits and improves neuromuscular junction (NMJ) morphology. Control NAP-treated and untreated larvae showed a homogeneous distribution of vesicular neuropeptide-Y-GFP (vGFP) fluorescence in peripheral nerves (b.i, a.i). Htau ${ }^{O N 3 R}$ larvae showed accumulation of vGFP, indicative of disrupted axonal transport $(\mathbf{c} . \mathbf{i}) .2 .5 \mu \mathrm{gml} \mathrm{m}^{-1} \mathrm{NAP}$ prevented accumulation of vGFP in htau ${ }^{\mathrm{ON} 3 \mathrm{R}}$ larvae (d.i). Larvae were reared at $23^{\circ} \mathrm{C}$. Scale bars: $10 \mu \mathrm{m}$. The total area encompassed by vGFP accumulates was greater in htau ${ }^{0 N 3 R}$ larvae compared with control. $2.5 \mu \mathrm{g} \mathrm{ml}^{-1}$ (low) and $25 \mu_{\mathrm{g} \mathrm{ml}}^{-1}$ (high) NAP reduced the area covered by vesicular accumulates back to control levels (e). Disruption of axonal transport was also evident in $A \beta_{42}$; htau $^{\text {ON4R }}$ and htau ${ }^{\text {ON4R }}$-expressing larvae when reared at $29^{\circ} \mathrm{C}$. NAP treatment significantly reduced the area covered by vGFP accumulates in both lines (f). NMJs on muscle 4 were revealed by anti-horseradish peroxidase (HRP) staining green (ii) and the tubulin cytoskeleton by anti-tubulin staining red (iii), overlay (iv). Control NAP-treated and untreated larvae exhibited a characteristic bead-like distribution of presynaptic boutons (b.ii, a.ii) and the tubulin cytoskeleton extended into the distal ends of the NMJ (b.iii, a.iii). Morphological aberrations (thinning of inter-bouton axon bundles and 'mini' satellite boutons) were seen in htau ${ }^{\mathrm{ON} 3 \mathrm{R}} \mathrm{NMJ}$ 's (arrowheads in c.ii). Anti-tubulin staining showed disruption of the cytoskeleton, with complete absence in the most distal regions of the NMJ (asterisk in c.iii). The NMJs of htau ${ }^{\text {ON3R }}$ NAP-treated animals contained a greater number of satellite boutons (arrowheads in d.ii) compared with untreated animals. However, restoration and extension of the cytoskeleton into the most distal ends of the NMJ was observed (asterisk in d.iii). Quantification of the area encompassed by NMJ's shows a significantly smaller NMJ area in htau ${ }^{\mathrm{ONBR}}$-expressing larvae when compared with controls. NAP treatment significantly increases the NMJ area in htau ${ }^{\mathrm{ON} 3 \mathrm{R}}$-expressing larvae $(\mathbf{g})$. Error bars represent mean \pm s.e.m., (e, g; one-way analysis of variance, post-hoc Bonferroni's multiple comparison test, $\left.{ }^{*} P<0.05 ;{ }^{*}{ }^{*} P<0.001 ;{ }^{*} * * P<0.0001\right)$, (f; unpaired Student's two-tailed $t$-test, $\left.{ }^{*} P<0.05 ;{ }^{*} P<0.01\right)$. $n=10(\mathbf{e}), n=5(\mathbf{f}), n=3-5(\mathbf{g})$.

bar Figure 5a). Furthermore, the rescue persisted even if htau ${ }^{\text {ON3R }}$ larvae reared on NAP for 4 days were taken off the drug and reared on normal food for a further 1 day (dark grey bar Figure 5a). Overall, the deficits observed at day 5 in all htau ${ }^{\mathrm{N} 3 \mathrm{R}}$ larvae reared on these variations of NAP treatment was significantly less than that observed in untreated htau ${ }^{\mathrm{N} 3 \mathrm{R}}$ larvae reared on normal food for 5 days (black bar Figure 5a). Interestingly, if NAP treatment was not begun at any point, the axonal transport disruption became progressively worse as the animals aged from day 4 to day 5 (first grey bar vs black bar in Figure $5 \mathrm{a}$ ).

To confirm the low toxicity of NAP, ${ }^{16,19,22}$ we assessed pupariation/ecclosion rates of control and htau ${ }^{\text {ON3R }}$ larvae with and without NAP treatment. NAP did not affect pupariation or ecclosion of either lines (Supplementary Figure 5). 


\section{DISCUSSION}

We demonstrate that compensating for tau loss-of-function by stabilising microtubules is a disease-modifying therapeutic strategy that should be considered for the treatment of tauopathies.
We show that treatment with the microtubule-stabilising peptide NAP significantly improves all phenotypes caused by expression of highly phosphorylated tau in vivo. This includes reinstatement of cytoskeletal integrity, prevention of axonal transport and synaptic a
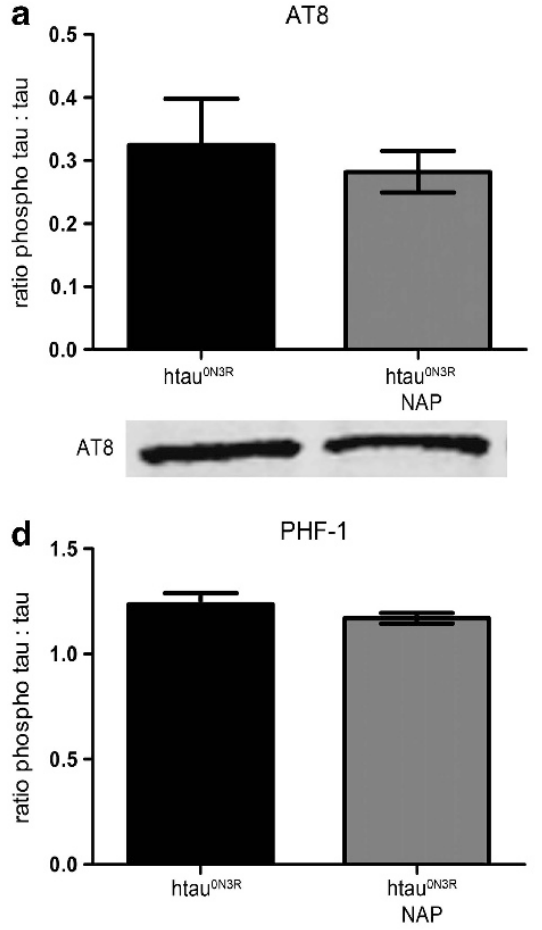

PHF-1
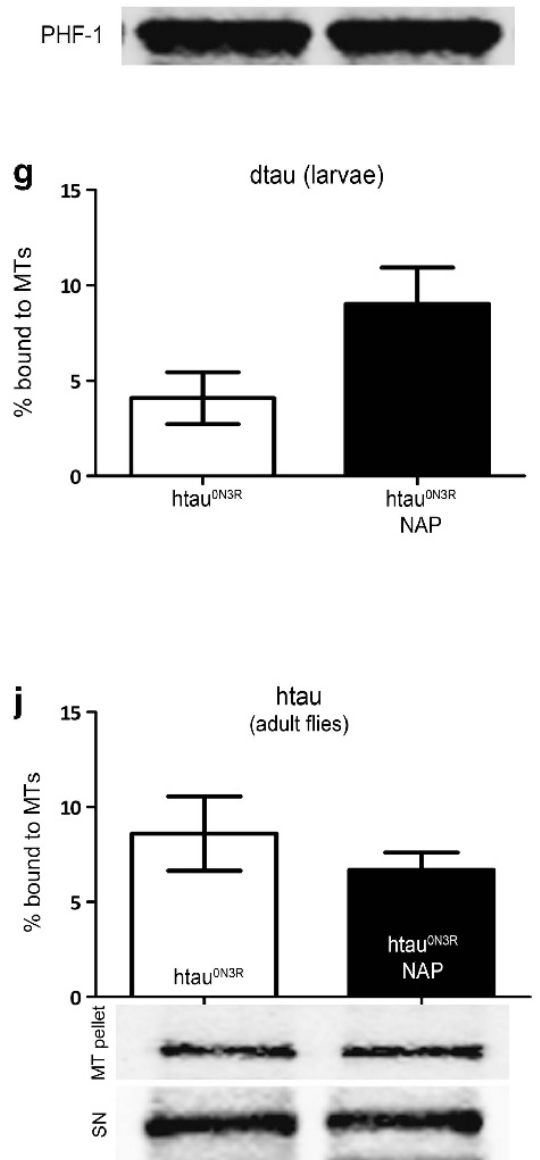

Figure 4. For caption please refer page 840 .
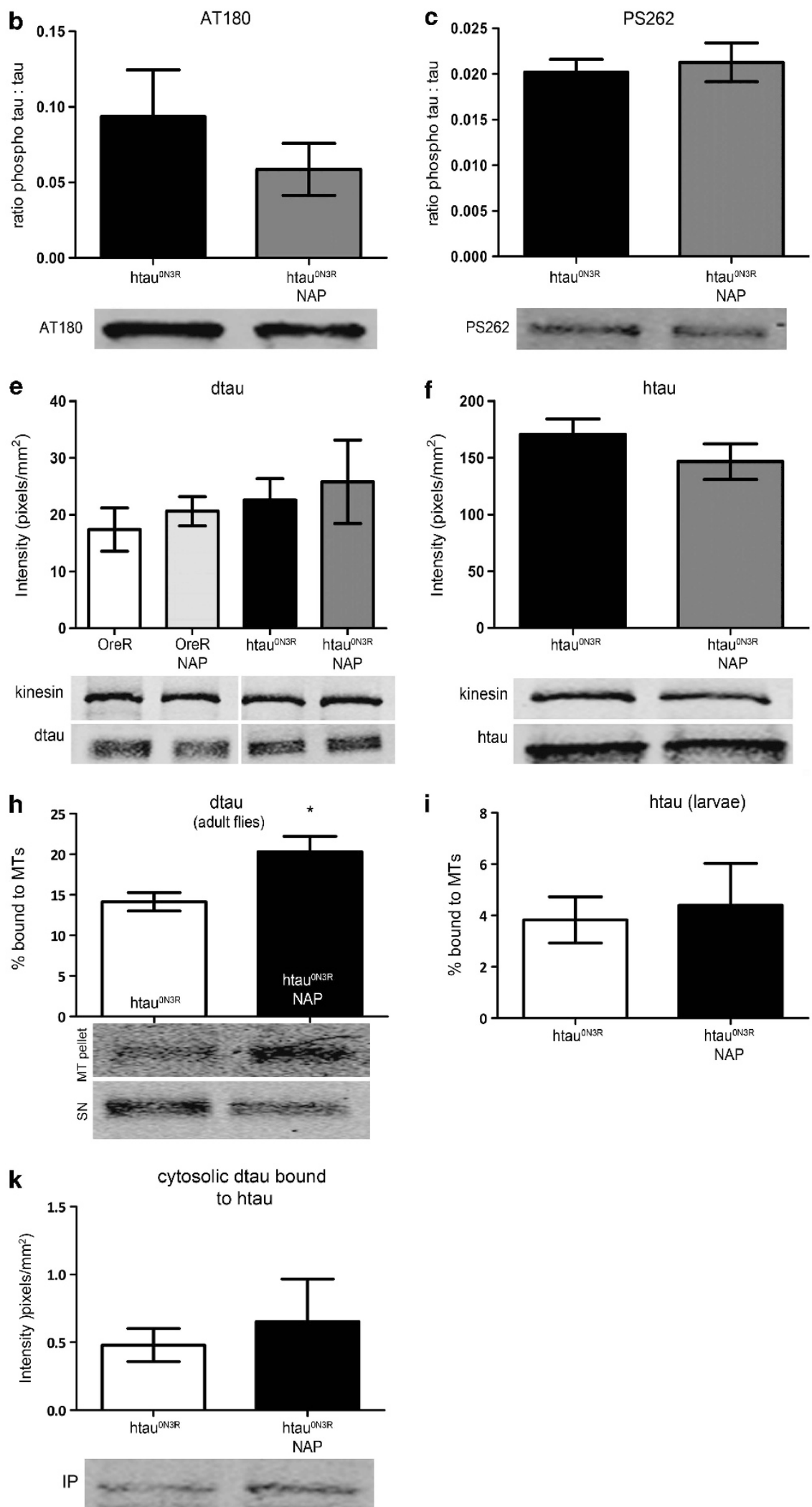
dysfunction and improvement of behavioural defects. Intriguingly, although hyper-phosphorylated tau is considered to be the toxic species in tauopathies, NAP rescued all phospho-tau phenotypes without altering phosphorylation at key disease-associated epitopes. Furthermore, NAP treatment is sufficient to rescue neuronal dysfunction even after it has already become established. Thus, NAP prevents, but more importantly, rescues phospho-tau-mediated neuronal dysfunction in vivo.

\section{NAP stabilises microtubules}

Microtubule integrity is critical for intracellular transport of materials and cytoskeletal dysfunction contributes to degeneration in many neurodegenerative conditions. Our previous findings illustrate that, microtubule destabilisation caused by tau loss-offunction is a key mechanism by which highly phosphorylated tau mediates neuronal dysfunction. ${ }^{4,6}$ In other models too, tau loss-offunction and subsequent trafficking impairments cause neurodegeneration. $^{25}$ The data presented here further underlines this and demonstrates that microtubule stabilisation can effectively protect against this.

Taxol-based microtubule-stabilising drugs are considered for treating $A D^{11,13,26,27}$ but their widespread toxicity and poor BBB penetrability precludes their use. To overcome this, a newer generation of drugs like small peptides (NAP) and small molecules (Epothilones, for example, EpoD) have been formulated. ${ }^{28-30}$ Both NAP and EpoD interact with MTs in vitro, reduce axonopathy and improve behaviour with minimal toxicity in rodent models of tauopathy. ${ }^{29,31}$ In agreement with these studies, we also report an improvement in behavioural deficits of htau ${ }^{\mathrm{ON} 3 \mathrm{R}}$-expressing larvae following treatment with NAP. However, we further extend these findings and demonstrate for the first time that re-instatement of the structure and function of microtubules is the mechanism by which NAP confers neuroprotection in vivo. Furthermore, our study pioneers evaluations of live axonal transport directly visualised in a living intact animal treated with NAP, or indeed any other microtubule-stabilising agent. Corroborating studies in rodents using $\mathrm{Mn}++$ enhanced magnetic resonance imaging are underway. ${ }^{32}$

We also observed that NAP restored the tubulin cytoskeleton within NMJs, and the resultant impact on synaptic function will have contributed to the behavioural improvements seen following NAP treatment. Furthermore, NAP has been shown to increase expression of the synaptic protein synaptophysin in neuronal cultures $^{33}$ and in vivo, ${ }^{34}$ which is associated with neuroprotection. ${ }^{35}$ However, the NMJs of NAP-treated htau ${ }^{\text {ON3R }}$ expressing animals had a greater number of 'mini' satellite boutons, which may reflect compensatory sprouting to maintain neuronal function. Indeed NAP has been shown to induce neurite outgrowth in vitro, ${ }^{17}$ and this would support our observations.

The molecular mechanism by which NAP stabilises MTs is not known. We hypothesise that NAP directly associates with MTs and modulates their dynamics. In support for this, NAP directly colocalises with tubulin ${ }^{17,36}$ and alters MT dynamics in cell culture. ${ }^{37}$ Our data also suggest that disorientated/misaligned MTs (such as those present in the htau-expressing larvae ${ }^{4}$ ) are NAP's most likely substrate, which is why it does not alter cytoskeletal integrity (and therefore neuronal function or behaviour) in controls. This is in complete agreement with recent studies showing a more robust effect on MT polymerisation in the compromised state. ${ }^{37,38}$

\section{NAP by-passes hyper-phosphorylated tau}

Clinical research in $A D$ has largely focused on anti-amyloid therapies, but the growing consensus now is that truly effective disease-modifying therapies will need to target tau as well. Thus, tau-based therapeutic targets are eagerly anticipated. Agents that reduce tau phosphorylation and aggregation are currently being tested in clinical trials. ${ }^{1,39}$ Interestingly, we find that microtubule stabilisation rescues neuronal dysfunction by by-passing hyperphosphorylated tau altogether and directly compensating for its detrimental effects. Similar results were reported in a rodent model of tauopathy in which EpoD improves cognitive performance without altering tau phosphorylation in young mice. ${ }^{29}$ However, in older mice with pre-existing tau pathology, EpoD reduces hyper-phosphorylated tau. ${ }^{40}$ Consistent with this, intranasal administration of NAP for 3 months reduces tau phosphorylated at AT8 and AT180 sites in triple transgenic AD mice. ${ }^{21}$ In contrast to these findings, we did not observe any significant changes in levels of either total tau or in phosphorylation of tau at the sites we examined following NAP treatment. This suggests that NAP is not affecting tau degradation or phosphorylation pathways. It is possible that in our model, where very early pre-tangle stages of disease are modelled and neuronal dysfunction before death is assessed, NAP-mediated protection occurs primarily due to microtubule stabilisation. However, it is conceivable that after prolonged periods of NAP treatment, as is the case in the studies described above, ${ }^{21,29,40}$ the restoration of microtubule integrity reinstates some of the homeostatic mechanisms (including some which impact tauphosphorylation) that may be lost in the presence of hyperphosphorylated tau. Our finding that NAP increases the percentage of dtau bound to MTs, which we previously showed is compromised by hyper-phosphorylated htau, ${ }^{4}$ supports this argument. We speculate that this occurs because stabilisation of MTs by NAP increases the MT surface area available for dtau binding, thus further improving MT integrity. As the level of dtau and the pathological dtau/htau interaction is unaltered (this is not surprising given that this interaction is dependent on the phosphorylation state of htau, ${ }^{4}$ which is unchanged by NAP), it is likely that dtau from the soluble cytosolic pool is recruited back to the MTs. Indeed dtau levels were reciprocally reduced in the supernatant fraction of NAP-treated animals.

\section{NAP rescues as well as prevents tau-mediated dysfunction}

An important consideration for therapies aiming to target dysfunctional cellular processes is the extent of damage that will have already occurred by the time clinical symptoms manifest. We therefore examined NAP's capacity to reinstate microtubule

Figure 4. NAPVSIPQ (NAP) does not alter tau phosphorylation at a number of sites relevant to Alzheimer's disease (AD); however, it increases dtau binding to the microtubules without altering the dtau/htau interaction. For each phospho-tau antigen, intensity of signal (pixels $\mathrm{mm}^{-2}$ ) was normalised to total tau. There was no significant change in the levels of the phospho-tau epitopes detected by AT8 (a), AT180 (b), PS262 (c) and PHF-1 (d) after treatment with NAP. Total dtau and htau levels were not altered by NAP treatment (e, f). Representative blots are shown below relevant graphs (a-f). Microtubule-binding assays were performed using larvae (g, i) or 1-to 3-day-old adult fly heads (h, j) expressing htau $^{\mathrm{ON} 3 \mathrm{R}}$ treated with or without $2.5 \mu \mathrm{g} \mathrm{ml}^{-1} \mathrm{NAP}$. Brain extracts were fractionated to yield a microtubule pellet (MT) and a soluble supernatant (SN). Both fractions were probed with anti-htau and anti-dtau antibodies. Treatment with NAP increased binding of endogenous dtau to the microtubules $(\mathbf{g}, \mathbf{h})$ but did not alter binding of htau to microtubules $(\mathbf{i}, \mathbf{j})$. Soluble human tau binds to Drosophila tau in a phosphorylationdependent manner. An anti-htau antibody was used to immunoprecipitate (IP) out htau from 1- to 3-day-old adult fly heads expressing htau ${ }^{\mathrm{ON} 3 \mathrm{R}}$, treated with or without $2.5 \mu \mathrm{g} \mathrm{ml}^{-1}$ NAP. Dtau co-immunoprecipitated with htau and NAP-treatment did not alter this interaction (k). All lanes were run on the same gel; however, (e) was non-contiguous. (Error bars represent mean \pm s.e.m., ${ }^{*} P<0.05$, unpaired Student's twotailed $t$ test, $n=5-7(\mathbf{e}-\mathbf{f}), n=3-5(\mathbf{g}-\mathbf{k}))$. 


\section{a}

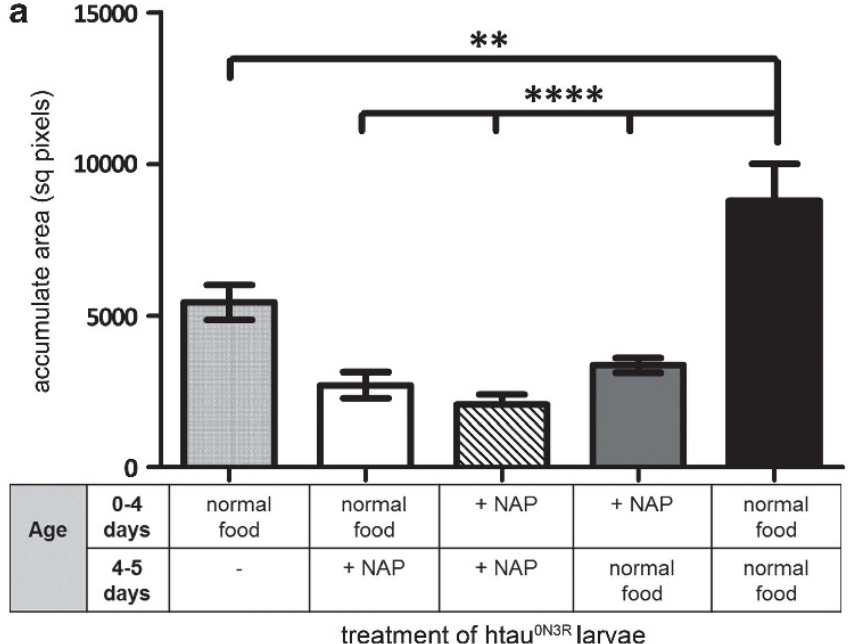

b

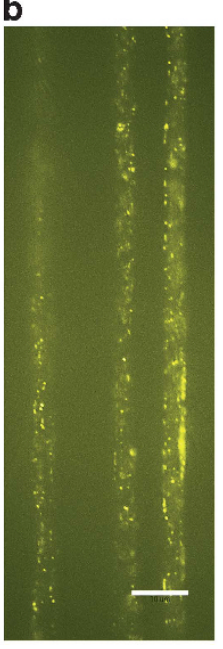

Figure 5. A $24 \mathrm{~h}$ NAPVSIPQ (NAP) treatment rescues the tau-mediated axonal transport phenotype. The total area encompassed by vesicular neuropeptide-Y-GFP (VGFP) accumulates was quantified over a pre-determined region of peripheral nerves in L3 larvae. A disruption of axonal transport was evident in untreated htau ${ }^{\mathrm{NN} 3 \mathrm{R}}$ larvae at day 4 (early L3) (light grey bar, far left) and became progressively worse by day 5 (black bar). However, NAP treatment for $24 \mathrm{~h}$ (white bar) significantly reduced the area covered by vesicular accumulates to the same level as that seen in larvae treated with NAP for 5 days (striped bar), thus rescuing the axonal transport deficit once established. NAP treatment for 4 days followed by transfer of larvae to normal food for $24 \mathrm{~h}$ was also sufficient to maintain protection at day 5 (dark grey bar) (a). Representative vGFP-tagged vesicle distribution in htau ${ }^{\mathrm{ON} 3 \mathrm{R}}, \mathrm{L} 3$ larval peripheral nerves treated for $24 \mathrm{~h}$ with $2.5 \mu \mathrm{g} \mathrm{ml}{ }^{-1}$ NAP (b). Scale bar: $10 \mu \mathrm{m}$. (Error bars represent mean \pm s.e.m., ${ }^{* *} P<0.01$; ${ }^{* * *} P<0.0001$, one-way analysis of variance, post-hoc Bonferroni's multiple comparison test, $n=10$ ).

integrity after it had already become compromised and found that treatment after onset of dysfunction was just as efficacious as prophylactic treatment before onset. This is supported by the findings of others that NAP is protective when administered immediately after injury ${ }^{18,41,42}$ and also when given chronically after the onset of dysfunction in rodent models of $A D .^{20,22,43}$ However, these studies have not investigated the effect of NAP treatment on MT integrity and function. Ours is the first study to investigate NAP's effects on both MT integrity (electron microscopy) and function (axonal transport) after the onset of dysfunction in vivo.

Furthermore NAP-mediated neuroprotection was long lasting, both in our model and in other models of neuronal injury. ${ }^{18,44}$ These results and ours are intriguing and suggest that, as speculated by Sudo and Bass, ${ }^{38}$ NAP may stabilise the microtubule lattice in such a way that its protective effect is retained, possibly like an 'imprint memory', even after its removal.

\section{CONCLUSION}

The mechanism(s) by which abnormally hyper-phosphorylated and aggregated tau cause neuronal dysfunction and neurodegeneration in tauopathies like AD is not entirely clear. The general consensus is that disease-associated aberrations (such as hyperphosphorylation in $A D$ and mutation in familial tauopathies), which reduce tau binding to microtubules cause cytoskeletal destabilisation leading to disruption of axonal transport before tau aggregation. Thus, microtubule stabilisation and restoration of axonal transport is probably the most promising disease-modifying therapeutic target to counter abnormal tau-mediated neuronal dysfunction. Our results support this view by demonstrating that a microtubule-stabilising drug can effectively protect against all hyper-phosphorylated tau-mediated phenotypes spanning from cellular and molecular dysfunction to behavioural defects in vivo. Microtubule-stabilising drugs such as NAP that penetrate the BBB and are non-toxic therefore hold great promise for the treatment of all tauopathies. Encouragingly phase II clinical trials showed promising results with improvements in cognitive scores, evidence of BBB permeability and good tolerability in patients with amnestic mild cognitive impairment. ${ }^{45}$ Therefore, NAP has been investigated in phase II/III clinical trials for the treatment of Progressive Supranuclear Palsy, a 'pure' severe tauopathy ${ }^{45}$ and the results of this are currently being evaluated. Understanding the mode of action of NAP will pave the way for similar disease-modifying therapies in $A D$ and related tauopathies that have thus far been untreatable.

\section{CONFLICT OF INTEREST}

The authors declare no conflict of interest.

\section{ACKNOWLEDGEMENTS}

This work was funded by the Alzheimer's Society with support from Henry Smith Charity and the Rosetrees Trust. We would like to thank Professor Illana Gozes (Tel Aviv University, Israel) for constructive comments.

\section{REFERENCES}

1 Bonda DJ, Lee HP, Lee HG, Friedlich AL, Perry G, Zhu X et al. Novel therapeutics for Alzheimer's disease: an update. Curr Opin Drug Discov Dev 2010; 13: 235-246.

2 Alonso AC, Zaidi T, Grundke-lqbal I, lqbal K. Role of abnormally phosphorylated tau in the breakdown of microtubules in Alzheimer disease. Proc Natl Acad Sci USA 1994; 91: 5562-5566.

3 Lovestone S, Reynolds $\mathrm{CH}$. The phosphorylation of tau: a critical stage in neurodevelopment and neurodegenerative processes. Neuroscience 1997; 78: 309-324.

4 Cowan CM, Bossing T, Page A, Shepherd D, Mudher A. Soluble hyper-phosphorylated tau causes microtubule breakdown and functionally compromises normal tau in vivo. Acta Neuropathol 2010; 120: 593-604.

5 Chee FC, Mudher A, Cuttle MF, Newman TA, MacKay D, Lovestone S et al. Over-expression of tau results in defective synaptic transmission in Drosophila neuromuscular junctions. Neurobiol Dis 2005; 20: 918-928.

6 Mudher A, Shepherd D, Newman TA, Mildren P, Jukes JP, Squire A et al. GSK-3beta inhibition reverses axonal transport defects and behavioural phenotypes in Drosophila. Mol Psychiatry 2004; 9: 522-530.

7 Terry RD. The pathogenesis of Alzheimer disease: an alternative to the amyloid hypothesis. J Neuropathol Exp Neurol 1996; 55: 1023-1025.

8 Cash AD, Aliev G, Siedlak SL, Nunomura A, Fujioka H, Zhu X et al. Microtubule reduction in Alzheimer's disease and aging is independent of tau filament formation. Am J Pathol 2003; 162: 1623-1627. 
9 Ishihara T, Hong M, Zhang B, Nakagawa Y, Lee MK, Trojanowski JQ et al. Age-dependent emergence and progression of a tauopathy in transgenic mice overexpressing the shortest human tau isoform. Neuron 1999; 24: 751-762.

10 Arriagada PV, Growdon JH, Hedley-Whyte ET, Hyman BT. Neurofibrillary tangles but not senile plaques parallel duration and severity of Alzheimer's disease. Neurology 1992; 42(3 Pt 1): 631-639.

11 Ballatore C, Brunden KR, Trojanowski JQ, Lee VM, Smith 3rd AB, Huryn DM Modulation of protein-protein interactions as a therapeutic strategy for the treatment of neurodegenerative tauopathies. Curr Top Medicinal Chem 2011; 11 317-330.

12 Gozes I. Tau pathology and future therapeutics. Curr Alzheimer Res 2010; 7: 685-696.

13 Trojanowski JQ, Smith AB, Huryn D, Lee VM. Microtubule-stabilising drugs for therapy of Alzheimer's disease and other neurodegenerative disorders with axonal transport impairments. Exp Opin Pharmacother 2005; 6: 683-686.

14 Zhang B, Maiti A, Shively S, Lakhani F, McDonald-Jones G, Bruce J et al. Microtubule-binding drugs offset tau sequestration by stabilizing microtubules and reversing fast axonal transport deficits in a tauopathy model. Proc Natl Acad Sci USA 2005; 102: 227-231.

15 Bassan M, Zamostiano R, Davidson A, Pinhasov A, Giladi E, Perl O et al. Complete sequence of a novel protein containing a femtomolar-activity-dependent neuroprotective peptide. J Neurochem 1999; 72: 1283-1293.

16 Gozes I, Morimoto BH, Tiong J, Fox A, Sutherland K, Dangoor D et al. NAP: research and development of a peptide derived from activity-dependent neuroprotective protein (ADNP). CNS Drug Rev 2005; 11: 353-368.

17 Divinski I, Holtser-Cochav M, Vulih-Schultzman I, Steingart RA, Gozes I. Peptide neuroprotection through specific interaction with brain tubulin. J Neurochem 2006; 98: 973-984.

18 Leker RR, Teichner A, Grigoriadis N, Ovadia H, Brenneman DE, Fridkin M et al. NAP, a femtomolar-acting peptide, protects the brain against ischemic injury by reducing apoptotic death. Stroke; J Cerebl Circ 2002; 33: 1085-1092.

19 Gozes I, Steingart RA, Spier AD. NAP mechanisms of neuroprotection. J Mol Neurosci 2004; 24: 67-72.

20 Shiryaev N, Jouroukhin Y, Giladi E, Polyzoidou E, Grigoriadis NC, Rosenmann H et al. NAP protects memory, increases soluble tau and reduces tau hyperphosphorylation in a tauopathy model. Neurobiol Dis 2009; 34: 381-388.

21 Matsuoka Y, Gray AJ, Hirata-Fukae C, Minami SS, Waterhouse EG, Mattson MP et al. Intranasal NAP administration reduces accumulation of amyloid peptide and tau hyperphosphorylation in a transgenic mouse model of Alzheimer's disease at early pathological stage. J Mol Neurosci 2007; 31: 165-170.

22 Matsuoka Y, Jouroukhin Y, Gray AJ, Ma L, Hirata-Fukae C, Li HF et al. A neuronal microtubule-interacting agent, NAPVSIPQ, reduces tau pathology and enhances cognitive function in a mouse model of Alzheimer's disease. J Pharmacol Exp Ther 2008; 325: 146-153.

23 Sinadinos C, Cowan CM, Wyttenbach A, Mudher A. Increased throughput assays of locomotor dysfunction in Drosophila larvae. J Neurosci Methods 2012 203: 325-334.

24 Sinadinos C, Burbidge-King T, Soh D, Thompson LM, Marsh JL, Wyttenbach A et al. Live axonal transport disruption by mutant huntingtin fragments in Drosophila motor neuron axons. Neurobiol Dis 2009; 34: 389-395.

25 Lei P, Ayton S, Finkelstein DI, Spoerri L, Ciccotosto GD, Wright DK et al. Tau deficiency induces parkinsonism with dementia by impairing APP-mediated iron export. Nat Med 2012; 18: 291-295.

26 Michaelis ML, Dobrowsky RT, Li G. Tau neurofibrillary pathology and microtubule stability. J Mol Neurosci 2002; 19: 289-293.

27 Michaelis ML, Chen Y, Hill S, Reiff E, Georg G, Rice A et al. Amyloid peptide toxicity and microtubule-stabilizing drugs. J Mol Neurosci 2002; 19: 101-105.

28 Brunden KR, Yao Y, Potuzak JS, Ferrer NI, Ballatore C, James MJ et al. The characterization of microtubule-stabilizing drugs as possible therapeutic agents for Alzheimer's disease and related tauopathies. Pharmacol Res 2011; 63: 341-351.
29 Brunden KR, Zhang B, Carroll J, Yao Y, Potuzak JS, Hogan AM et al. Epothilone D improves microtubule density, axonal integrity, and cognition in a transgenic mouse model of tauopathy. J Neurosci 2010; 30: 13861-13866.

30 Gozes I, Divinski I. NAP, a neuroprotective drug candidate in clinical trials, stimulates microtubule assembly in the living cell. Curr Alzheimer Res 2007; 4 507-509.

31 Barten DM, Fanara P, Andorfer C, Hoque N, Wong PY, Husted KH et al. Hyperdynamic microtubules, cognitive deficits, and pathology are improved in tau transgenic mice with low doses of the microtubule-stabilizing agent BMS-241027. J Neurosci 2012; 32: 7137-7145.

32 Davunetide (NAP) and D-NAP (AL-408) protect against tau hyperphosphorylation in a model of ALS: implications for axonal transport. Neuroscience Meeting Planner. Society for Neuroscience: New Orleans, LA, USA, 2012, abstract 242.05/E23.

33 Smith-Swintosky VL, Gozes I, Brenneman DE, D'Andrea MR, Plata-Salaman CR. Activity-dependent neurotrophic factor-9 and NAP promote neurite outgrowth in rat hippocampal and cortical cultures. J Mol Neurosci 2005; 25: 225-238.

34 Idan-Feldman A, Schirer Y, Polyzoidou E, Touloumi O, Lagoudaki R, Grigoriadis NC et al. Davunetide (NAP) as a preventative treatment for central nervous system complications in a diabetes rat model. Neurobiol Dis 2011; 44: 327-339.

35 Pascual M, Guerri C. The peptide NAP promotes neuronal growth and differentiation through extracellular signal-regulated protein kinase and Akt pathways, and protects neurons co-cultured with astrocytes damaged by ethanol. J Neurochem 2007; 103: 557-568.

36 Divinski I, Mittelman L, Gozes I. A femtomolar acting octapeptide interacts with tubulin and protects astrocytes against zinc intoxication. J Biol Chem 2004; 279 28531-28538.

$37 \mathrm{Oz} \mathrm{S}$, Ivashko-Pachima Y, Gozes I. The ADNP derived peptide, NAP modulates the tubulin pool: implication for neurotrophic and neuroprotective activities. PloS One 2012; 7: e51458.

38 Sudo H, Baas PW. Strategies for diminishing katanin-based loss of microtubules in tauopathic neurodegenerative diseases. Hum Mol Genet 2011; 20: 763-778.

39 Mangialasche F, Solomon A, Winblad B, Mecocci P, Kivipelto M. Alzheimer's disease: clinical trials and drug development. Lancet Neurol 2010; 9: 702-716.

40 Zhang B, Carroll J, Trojanowski JQ, Yao Y, Iba M, Potuzak JS et al. The microtubulestabilizing agent, epothilone $D$, reduces axonal dysfunction, neurotoxicity, cognitive deficits, and Alzheimer-like pathology in an interventional study with aged tau transgenic mice. J Neurosci 2012; 32: 3601-3611.

41 Beni-Adani L, Gozes I, Cohen Y, Assaf Y, Steingart RA, Brenneman DE et al. A peptide derived from activity-dependent neuroprotective protein (ADNP) ameliorates injury response in closed head injury in mice. J Pharmacol Exp Ther 2001; 296: 57-63.

42 Rotstein M, Bassan H, Kariv N, Speiser Z, Harel S, Gozes I. NAP enhances neurodevelopment of newborn apolipoprotein E-deficient mice subjected to hypoxia. J Pharmacol Exp Ther 2006; 319: 332-339.

43 Vulih-Shultzman I, Pinhasov A, Mandel S, Grigoriadis N, Touloumi O, Pittel Z et al. Activity-dependent neuroprotective protein snippet NAP reduces tau hyperphosphorylation and enhances learning in a novel transgenic mouse model. J Pharmacol Exp Ther 2007; 323: 438-449.

44 Jehle T, Dimitriu C, Auer S, Knoth R, Vidal-Sanz M, Gozes I et al. The neuropeptide NAP provides neuroprotection against retinal ganglion cell damage after retinal ischemia and optic nerve crush. Graefe's archive for clinical and experimental ophthalmology $=$ Albrecht von Graefes Archiv fur klinische und experimentelle Ophthalmologie 2008; 246: 1255-1263.

45 Gold M, Lorenzl S, Stewart AJ, Morimoto BH, Williams DR, Gozes I. Critical appraisal of the role of davunetide in the treatment of progressive supranuclear palsy. Neuropsychiatric Disease Treatment 2012; 8: 85-93.

(c) (i) $\Theta$ This work is licensed under a Creative Commons AttributionNonCommercial-NoDerivs 3.0 Unported License. To view a copy of this license, visit http://creativecommons.org/licenses/by-nc-nd/3.0/

Supplementary Information accompanies the paper on the Molecular Psychiatry website (http://www.nature.com/mp) 